\title{
Brian Josephson: 80th Birthday
}

\section{Francesco Tafuri $^{1}$}

Published online: 4 March 2019

(C) Springer Science+Business Media, LLC, part of Springer Nature 2020

I have been asked by the Editorial Board to take care of a short note in honor of Prof. Brian Josephson who turned 80 on Jan. 4, 2020. This is not an easy task, because I have no direct personal memories to tell, but it is made possible by the awareness that my scientific life has been better and happier because of the Josephson effect [1,2]. The idea that Cooper pairs could coherently tunnel through a barrier offering a direct way to measure, control, and manipulate the phase difference of the macroscopic wave functions of the two superconductors at the sides of the junction has been of inspiration for a lot of scientists in different fields of research activities.

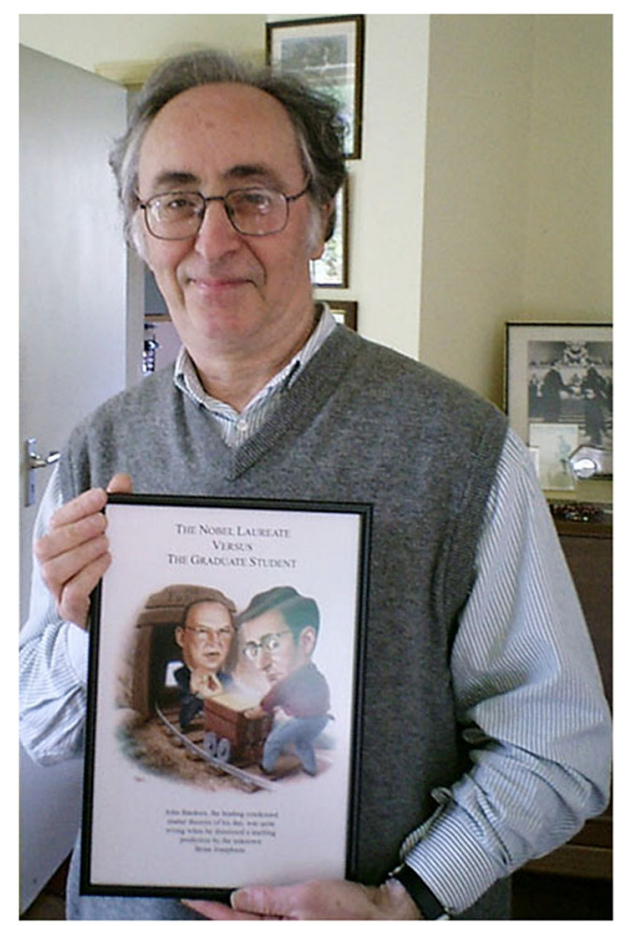

Francesco Tafuri

francesco.tafuri@unina.it

1 Università di Napoli Federico II, Napoli, Italy
Josephson was born in Cardiff and in 1957 he moved to Cambridge, where he initially studied Mathematics at Trinity College. After two years, he switched to Physics. He graduated in 1960 and became a research student in the university's Mond Laboratory on the old Cavendish site, under the supervision of Brian Pippard. It was during this period, as a $\mathrm{PhD}$ student in 1962, that he carried out the research that led to his discovery of the Josephson effect. He was elected a fellow of Trinity College in 1962, and obtained his PhD in 1964 for a thesis entitled Non-linear conduction in superconductors.

Josephson was 22 years old when he did the work on quantum tunneling that won him the Nobel Prize. He discovered that a supercurrent could tunnel through a thin barrier, predicting the presence of a current flow with no drop in voltage, which depends on the magnetic field. In presence of a voltage drop, the current should oscillate at a frequency related to the drop in voltage. This became known as the Josephson effect and the device as a Josephson junction. His calculations were published in Physics Letters in a paper entitled Possible new effects in superconductive tunneling, received on 8 June 1962 and published on 1 July [1]. His original paper was published before writing his fellowship thesis.

A beautiful account of the scientific history of the discovery is given by Kristian Fossheim in his book Superconductivity: Discoveries and Discoverers [3]. The paper by Anderson on the pseudo-spin model for superconductivity with analogies with magnetism was inspiring for Josephson to search conditions under which phase of the wave function would matter. Josephson credits Phil Anderson with the insight he had gained in the concept of broken symmetry. Anderson was in Cambridge at that time and giving a course on concepts in solids. Josephson found that the phase could only matter if there was a transfer of Cooper pairs through the contact. This motivated him to extend the method by Cohen, Falicov, and Philips, who had a way of calculating the tunneling current, to the case of junctions with superconducting electrodes. This gave the phase difference-dependent factor, which he was expecting, and also the zero voltage current, which was not necessarily expected. The idea of the phase as something that would occur in a tunneling experiment was the original inner 
motivation of the discovery. Pippard did not have much faith in the phase as an important aspect. So Josephson felt important the support from Anderson, because he encouraged him and said "That seems to be right." The flux-quantization experiments by Deaver and Fairbanks around 1960 was the main support at that time that the phases were a "real thing." The effects were confirmed experimentally by Philip Anderson and John Rowell of Bell Labs in Princeton, as reported in their paper, Probable Observation of the Josephson Superconducting Tunneling Effect (Physical Review Letters [4]). As for ac Josephson effect, it has been observed by Yanson et al. [5].

In the meanwhile when Josephson's paper came out, Bardeen's attitude was negative. Details on the debate can be found in Fossheim's book, starting from the first public discussion at Queen Mary College in London at a lowtemperature conference. Bardeen had this-in Josephson's mind-erroneus argument that pairing would not continue through the barrier, causing decoherence. A few months later, Bardeen made a speech saying Josephson was right. The situation with "the student against the Nobel laureate" became a famous story. It entered the pages of Physics Today.

Josephson was awarded several important prizes for his discovery, including the 1969 Research Corporation Award for outstanding contributions to science, and the Hughes Medal and Holweck Prize in 1972. In 1973, he won the Nobel Prize in Physics, sharing the award with two other scientists who had also worked on quantum tunneling. Josephson was awarded half the prize "for his theoretical predictions of the properties of a supercurrent through a tunnel barrier, in particular those phenomena which are generally known as the Josephson effects."

The other half of the award was shared equally by Japanese physicist Leo Esaki of the Thomas Watson Research Center in Yorktown, New York, and Norwegian-American physicist Ivar Giaever of General Electric in Schenectady, New York, "for their experimental discoveries regarding tunneling phenomena in semiconductors and superconductors, respectively."

Josephson spent a postdoctoral year in the USA (19651966) as research assistant professor at the University of
Illinois at Urbana-Champaign. After returning to Cambridge, he was made assistant director of research at the Cavendish Laboratory in 1967, where he remained a member of the Theory of Condensed Matter group, a theoretical physics group, for the rest of his career. In 1972, he became a reader in physics at Cambridge and in 1974 a full professor, a position he held until he retired in 2007.

The Editorial Board of the Journal of Superconductivity and Novel Magnetism has no better way to wish happy birthday than saying, Thank you, Prof. Josephson! Happy Birthday! "Your effect" has made our scientific life more interesting and exciting. We can see in it the elegance of quantum mechanics, and all quantum devices based on your discovery are an amazing confirm of its power.

Much has to be expected in the future. The horizons of a system, which keeps coherence on a macroscopic scale, can sustain measurable macroscopic quantum phenomena, and can be eventually tuned at the nanoscale, are extremely wide and forever young [6-9].

Acknowledgements Pleasant conversations on the topic with $\mathrm{H}$. Fossheim and V. Kresin are gratefully acknowledged.

\section{References}

1. Josephson, B.: Phys. Lett. 7, 251 (1962)

2. Josephson, B.: In: Parks, R. (ed.) Superconductivity, vol. 1, p. 423 (1969)

3. Fossheim C: Superconductivity: Discoveries and Discoverers. Springer Nature, Switzerland (2013)

4. Anderson, P., Rowell, R.: Phys. Rev. Lett. 10, 230 (1963)

5. Yanson, I., Svistunov, V., Dmitrenko, I.: JETP. 20, 1404 (1965)

6. Barone, A., Paterno, G.: Physics and Applications of the Josephson Effect. John Wiley, New York (1982)

7. Likharev K.K.: Dynamics of Josephson Junctions and Circuits. Gordon and Breach, New York (1986)

8. Braginski, A.: J. Super. Novel Magn. 32, 23 (2019)

9. Tafuri, F. (ed.): Fundamentals and Frontiers of the Josephson Effect. Springer Nature, Switzerland (2019)

Publisher's note Springer Nature remains neutral with regard to jurisdictional claims in published maps and institutional affiliations. 